\title{
AERIAL FOGGY IMAGE REJECTION USING SINGLE NEURON
}

\author{
Mihaela Bukljaš', Ivan Pavić2, Pero Vidan², Srđan Vukša ${ }^{2 *}$ \\ 1 University of Zagreb, Faculty of Transport and Traffic science, Zagreb, Croatia \\ ${ }^{2}$ University of Split , Faculty of Maritime Studies, Split, Croatia
}

Aerial images taken during terrain mapping can be affected with a foggy weather. In this paper we proposed new fog detection method and image rejection using single neuron. Fog detection is based on statistical data collected by converting color image to grayscale image and then applying gamma correction factor. With this statistical data, training dataset is created for single neuron. Neuron is simple two input neuron with sigmoid activation function. We establish connection with single neuron and statistical data so it can recognize is there presence of fog in aerial image.

Key words: single neuron network, foggy aerial image, UAV

\section{INTRODUCTION}

Clouds are visible aggregate of minute particles of water or ice, or both in the free air [1]. Since clouds take place at sky their presence can be anywhere on the planet at any time. If we want to take some aerial images of terrain then clouds can present an obstacle which will affect poor terrain visibility in image.Today more and more unmanned aerial vehicles (UAV) are used in every day application, especially in missions of Search and Rescue (SAR) [2]. For great importance it is necessary to know the terrain so SAR can be doable. First we have to map the terrain as best as we can, and today the best approach for that is taking aerial images with UAV's. Since mapping will be conduct on good weather condition, there is always a chance for clouds to appear, which can affect on poor terrain visibility, also the image quality can be affected by different factors such as camera type, terrain characteristics, etc. [3]. From the UAV's point of view cloud can be seen as cloud or as fog depending on UAV's position. In this paper research is made on assumption that UAV's is flying through the cloud so the problem is represented as fog. Since UAV's are less computer powerful it is challenge to implement simple and effective algorithm that will detect foggy aerial image and decide should it be rejected or kept for terrain mapping.

The main purpose of this research is to find simple, less computer demanding method that will manage to fulfil that challenge. Our approach is based on statistical data obtained from converting color image to grayscale image and applying gamma correction factor to specific value. With some aerial images we have created training dataset for neuron. Neuron is simple two input neuron with sigmoid activation function so we can easily recognize should image be rejected or not. In chapter 2 . It will be discussion about other methods used for fog detection within images, many of the approaches are related to fog detection in vehicles. Our approach of fog detection in aerial images is described in chapter 3 . Results obtained by our method are described in chapter 4 .

\section{RELATED WORK}

There are many methods of detecting fog within image, many of them are related to land vehicles. In the past year fog detection based on image processing has been studied so the fog effect can be removed from image [4]. Proposed method by Narasimhan and Nayar [5] is based on restoring contrast of the original image. Since image is degraded due to atmospheric conditions they develop a new physics-based model for multiple scattering of light rays as they travel from a source to an observer where weather condition and visibility of the atmosphere can be estimated. Tan [6] proposes several methods on image fog removal, first of them exploit two or more images of the same scene with different degrees of polarization. Second one is to use multiple images with bad weather conditions and exploit the differences of the images with same scene that have different properties of participating medium. Third approach is based on single image and $3 \mathrm{D}$ geometrical model of the input scene but main drawback of all these methods is that they cannot be applied to dynamic scenes but is able to restore image color and gray scale. In [7] He et al. proposed dark channel prior method on color image to detect haze and restore the image. New extended algorithm for fog detection is proposed by Tarel et al. [8] which better handles road images where large part of image can be assumed to be a planar road. Wang [3] proposed road extraction from aerial foggy image where image is defogging method based on modified dark channel prior is applied so contrast and highlight of road areas can be more visible. Mao et. al. [10] proposed a function for estimating the haze degree for the automatic detection of the foggy image with different haze degrees. New approach for detection of foggy images, together with dehazing, has been proposed by applying deep neural network [9][11][12] .

\section{OUR METHOD}

\section{Single neuron}

Since idea was to make everything simple as possible, we have created one single neuron shown in figure 1. 


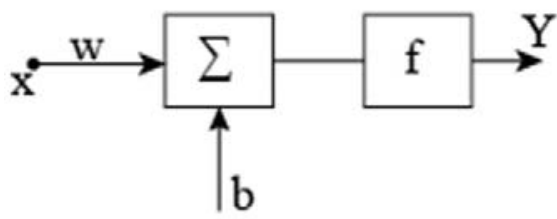

Figure 1: Representation of single neuron architecture

Where input $x$ is the ratio between grayscale image mean and standard deviation. Weight $w$ and bias $b$ are normally distributed random numbers. There are several activation functions fi.e. step, linear, tangh, sigmoid, ReL Uect. for this neuron sigmoid activation function was used since output range will always be in range $(0,1)$. Since small amount of fog in images is not of concern to visibility of image sigmoid activation function shown in equation (1) will tend to respond very less to changes in input $x$.

$\operatorname{sigmoid}(\alpha)=\frac{1}{1+e^{-\alpha}}$

Output of neuron $Y$ can be expressed by following equation (2).

$Y=\operatorname{sigmoid}\left(\sum(w \cdot x)+b\right)$

\section{Fog detection}

Our approach to fog detection in image is based on grayscale image enhanced with gamma correction factor in a way that intensity of a light at each pixel is more significant than simple grayscale image. By applying gamma correction factor on a foggy grayscale image, ratio between mean and standard deviation of pixels is taken. With this ratio there is good approximation of images that should be kept or rejected due to poor visibility. Value of gamma correction factor is taken randomly in a way where ratio presented in equation (3), between mean and standard deviation will distinguished image visibility and classify it as positive or negative sample (1 or 0 ).

ratio $=\frac{\text { mean }}{\text { std.deviation }}$

In table 1. ratio of mean and standard deviation is taken from same set of images and therefore classify them by good or bad visibility.

Table 1: Image visibility classification by gamma correction factor and ratio of mean and standard deviation

\begin{tabular}{|c|c|c|c|c|c|c|c|}
\hline \multirow{2}{*}{ gamma } & \multicolumn{7}{|c|}{ Image ratio } \\
\cline { 2 - 8 } & 1 & 2 & 3 & 4 & 5 & 6 & 7 \\
\hline 1 & 2,95 & 2,26 & 2,33 & 4,86 & 3,45 & 2,37 & 2,60 \\
\hline 1.5 & 2,38 & 1,84 & 1,848 & 3,95 & 2,92 & 2,02 & 2,17 \\
\hline 2 & 1,76 & 1,40 & 1,33 & 2,97 & 2,36 & 1,66 & 1,70 \\
\hline 2.5 & 1,11 & 0,97 & 0,84 & 1,97 & 1,79 & 1,28 & 1,18 \\
\hline visibility & 1 & 1 & 1 & 0 & 0 & 0 & 0 \\
\hline
\end{tabular}

From table 1. it can be seen that ratio of images 6 and 7 with gamma correction factor less than 2.5 classifies images as good visibility which is not desirable, therefore gamma correction factor 2.5 is used. Steps of our fog detection algorithm are presented in figure 2. We start by taking picture with camera. This input image is then converted to grayscale image. Next step is to add gamma correction factor of 2.5 to image and calculate ration between mean and standard deviation of image. This ratio is the input of the trained neuron which will decide to keep or reject image.

\section{The automatic image classification}

The proposed fog detection algorithm is implemented in MATLAB using image processing toolbox. Single neuron design is also implemented through the same software. The experimented images are taken from video:

(hhtp://www.youtube.com/watch?v=GfxdeR2fLA\&t=1s).

From this videos 15 images are taken for training neuron. Images have been hand classified as positive or negative samples, 6 of them are positive while rest as negative samples. Using MATLAB function to convert RGB image to grayscale we have converted all 15 samples. Next gamma correction factor of 2.5 is applied to all images and MATLAB function for image mean and standard deviation is used.

Figure 3. show the implementation of grayscale function and gamma correction factor. Calculated values of image mean and standard deviation are plotted so we can see good classification of positive and negative samples. Figure 4. show plotted graph of 15 images used for neuron training. After we have collected data set we start to train neuron. Learning rate is set experimentally to 0.1

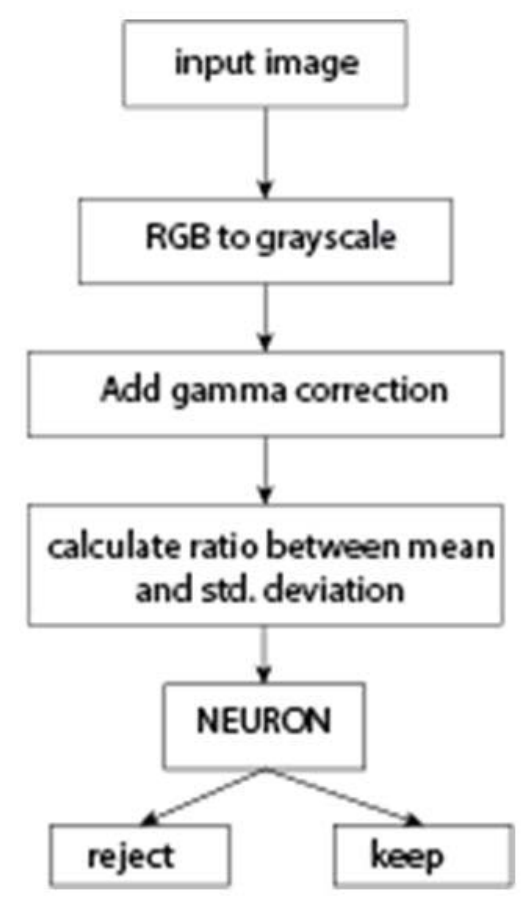

Figure 2: Fog detection architecture

Istrazivanja i projektovanja za privredu ISSN 1451-4117 Journal of Applied Engineering Science Vol. 18, No. 2, 2020 
and training rate is set to 1000 iteration where value of weight $w$ and bias $b$ is calculated. To evaluate our method of fog detection we have tested it with 20 more images extracted from the same video. Results are presented in figure 5.

\section{CONCLUSION}

In this paper we have presented a new method for fog detection in aerial images, inspired by many traditional defogging methods using convolution neural network (CNN) and statistical data of image with dark channel prior method. Our method uses only algorithm and simple neuron so the fog can be detected. Main advantage of this method is speed and low computational power which will suit for every drone. However, this method is tested only with specific aerial images so more research has to be done. Since there are no many aerial images with foggy weather our next goal is to add synthetic fog to aerial images and expend training dataset. After sufficient number of data we collect this method will be implemented on JETSON TK1 development kit used in drones so the live testing can be done.

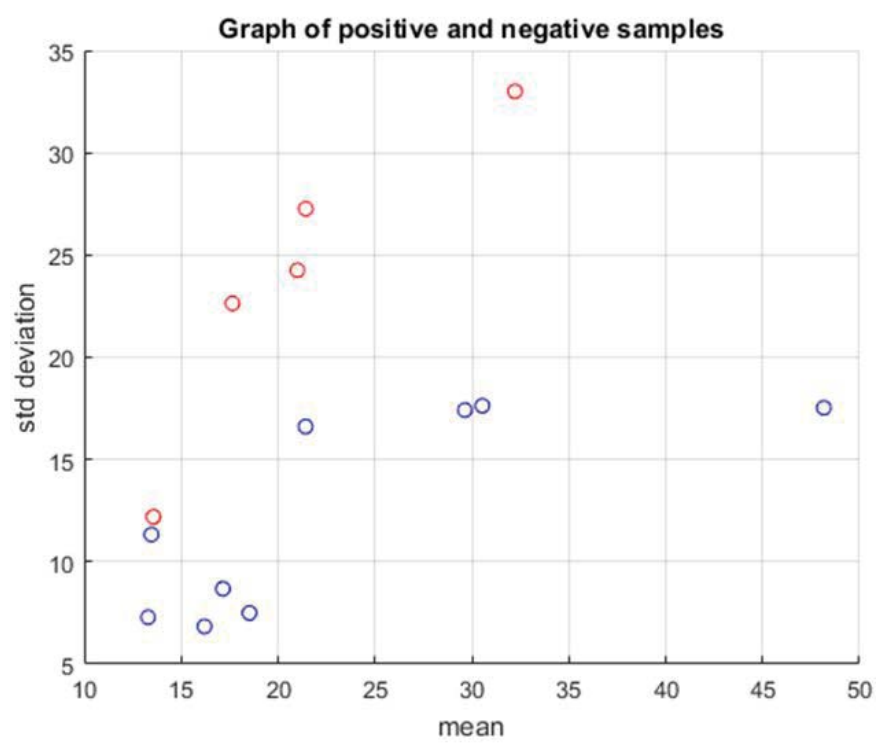

Figure 4: positive samples (red), negative samples (blue)

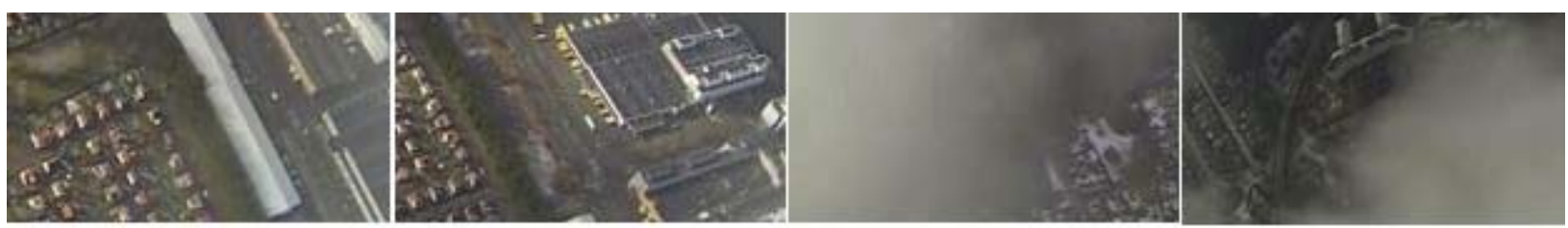

(a)

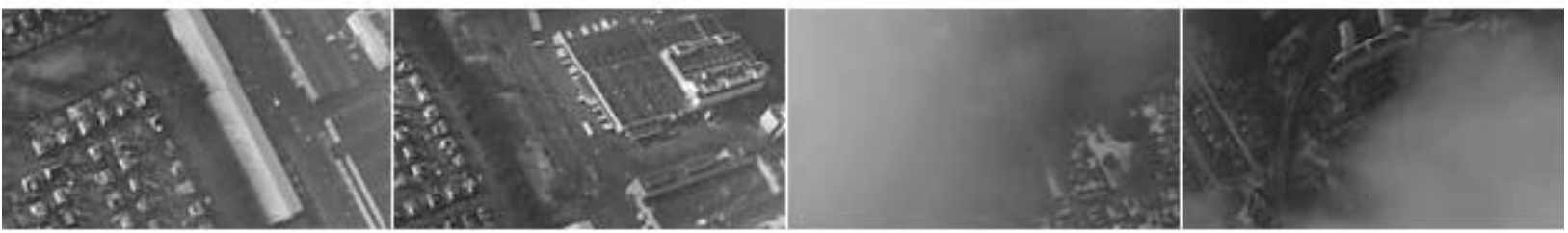

(b)

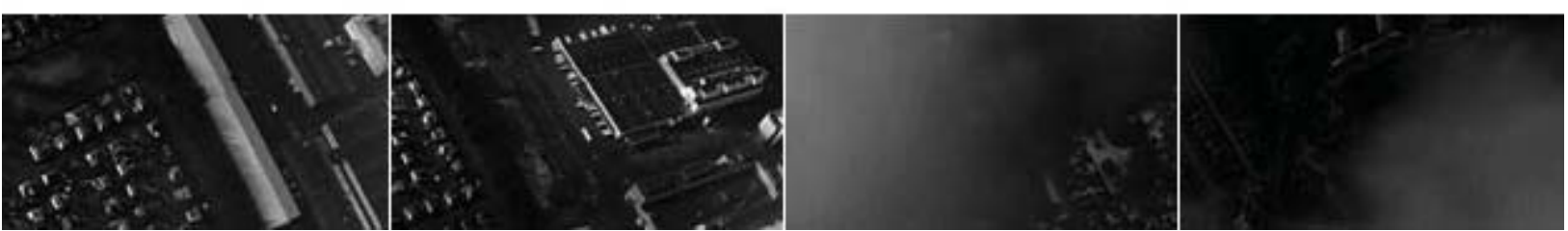

(c)

Figure 3: (a) input image, (b) grayscaleconversion, (c) appliedgammacorrection
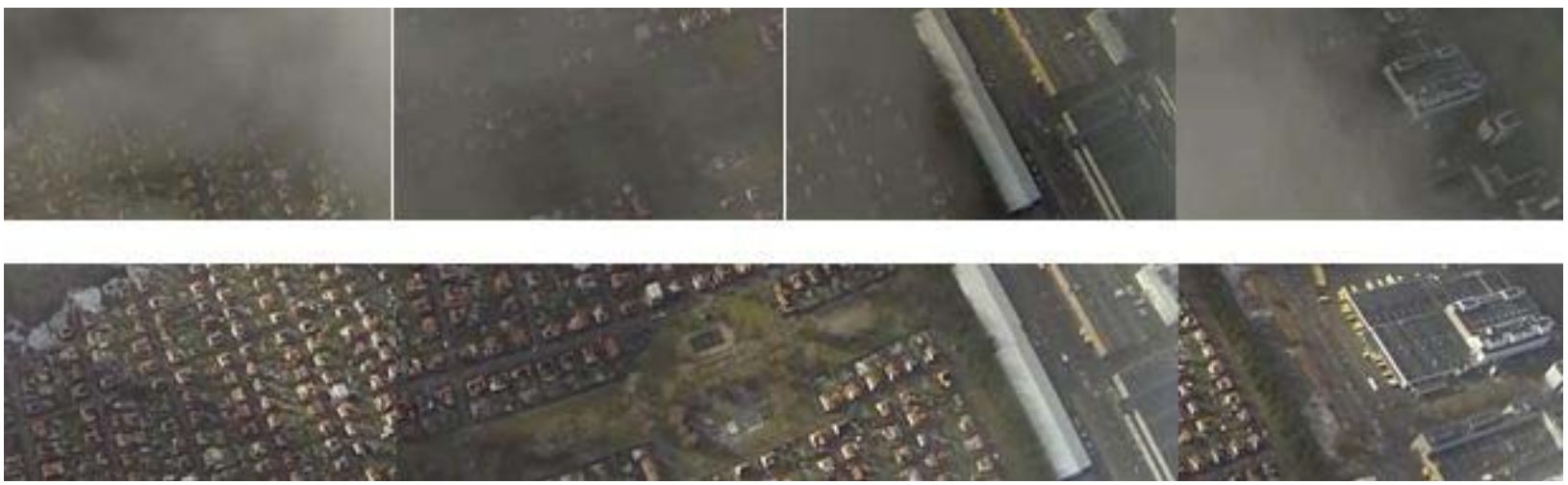

Figure 5: (a) rejected images, (b) kept images 


\section{REFERENCES}

1. Robert A. Houze, Jr. "Cloud Dynamics", Academic Press, In. 1250 Sixth Avenue, San Diego, California 92101-4311 p. xiii, 1994.

2. S. Waharte and N. Trigoni, "Supporting search and rescue operations with UAVs," in Proceedings - EST 2010 - 2010 International Conference on Emerging Security Technologies, ROBOSEC 2010 - Robots and Security, LAB-RS 2010 - Learning and Adaptive Behavior in Robotic Systems, 2010.

3. Wang F. Wang W. "Road extraction using modified dark channel prior and neighborhood FCM in foggy aerial images", Springer Science+Bussines Media, LLC, part of Springer Nature 2018.

4. Negru M. Nedveschi S. "Image Based Fog Detection and Visibility Estimation for Driving Assistance Systems" 2013 IEEE 9th International Conference on Intelligent Computer Communication and Processing (ICCP), 2013.

5. S. G. Narasimhan, S. K. Nayar, "Shedding light on the weather. In Proceedings of the 2003 IEEE computer society conference on Computer vision and pattern recognition (CVPR'03). IEEE Computer Society, Washington, DC, USA, pp. 665-672.

6. Tan, R.T., "Visibility in bad weather from a single image", IEEE Conference on Computer Vision and Pattern Recognition, 2008. CVPR 2008, pp.1-8, 2328 June 2008.
7. Kaiming He, Jian Sun, Xiaoou Tang, "Single image haze removal using dark channel prior," IEEE Conference on Computer Vision and Pattern Recognition, 2009. CVPR 2009, pp.1956-1963, 20-25 June 2009.

8. Tarel, J-P., Hauticre, N., Cord, A., Gruyer, D., Halmaoui, H., "Improved visibility of road scene images under heterogeneous fog," Intelligent Vehicles Symposium (IV), 2010 IEEE, pp.478-485, 21-24 June 2010.

9. G. Pagani, W. Wauben, J.W. Noteboom, „Neural network approach for automatic fog detection using surveillance camera images" Geophysical esearch Abstracts Vol. 20, EGU2018-2988, 2018

10. J. Mao, U. Phommasak, S. Watanabe, H. Shioya, "Detecting Foggy images and Estimating the Haze Degree Factor", Mao et al., J ComputSciSystBiol 2014.

11. S. Mengyun, X. Fengying, Z. Yue, Y. Jihao, "Cloud detection of remote sensing images by deep learning" 2016 IEEE International Geoscience and Remote Sensing Symposium (IGARSS)

12. C. Bolun, X. Xiangmin, J. Kui, Q. Chunmei, T. Dacheng, "Dehaze Net: An End-to-End System for Single Image Haze Removal", Computer Vision and Pattern Recognition (cs.CV) 2016. 